\title{
Variation of magnetic easy direction of bcc structured ferromagnetic films up to thirty spin layers
}

\author{
N.U.S. Yapa ${ }^{2,3}$, P. Samarasekara ${ }^{1,3^{*}}$ and Sunil Dehipawala ${ }^{4}$ \\ ${ }^{I}$ Department of Physics, University of Peradeniya, Peradeniya, Sri Lanka \\ ${ }^{2}$ Department of Physics, Open University of Sri Lanka, Kandy, Sri Lanka \\ ${ }^{3}$ Postgraduate Institute of Science, University of Peradeniya, Sri Lanka \\ ${ }^{4}$ Physics Department, Queensborough Community College of CUNY, 222-05, 56th Avenue, Bayside, NY 11364, \\ USA
}

\begin{abstract}
Third order Heisenberg Hamiltonian was employed to determine the effect of number of spin layers on magnetic easy direction. 3-D graph of energy versus stress induced anisotropy and angle was plotted to find magnetic easy and hard directions. First values of stress induced anisotropy corresponding to minima and maxima of this 3-D plot were determined. Then the graph of energy versus angle was plotted at each of these stress induced anisotropy values in order to determine magnetic easy and hard directions. The same approach was applied to find the easy and hard directions for different values of spin exchange anisotropy. Magnetic easy direction gradually rotates from out of plane to in plane direction of the film, as the number of spin layers is increased from 10 to 30 . The magnetic anisotropy energy (energy difference between magnetic easy and hard directions) gradually increases as the number of layers is increased. However, the angle between easy and hard directions doesn't change considerably in this range of thickness. Our theoretical data agree with experimental data obtained by some other researchers. Easy axis oriented magnetic thin films are useful in magnetic memory devices due to the higher magnetic anisotropy and higher energy density.
\end{abstract}

Keywords: Heisenberg Hamiltonian, third order perturbation, magnetic easy axis, spin layers.

\section{INTRODUCTION}

Ferromagnetic thin films are prime candidates in magnetic memory and microwave devices. The second order perturbed Heisenberg Hamiltonian was previously applied to investigate the magnetic properties of ferromagnetic films with four and five spin layers by us (Samarasekara 2008a, Samarasekara and Warnakulasooriya 2016). In second order perturbed Heisenberg Hamiltonian, terms with second order of $\varepsilon$ were considered. In first manuscript, second order perturbed Heisenberg Hamiltonian of ferromagnetic films with four layers was considered under special assumptions to avoid tedious derivations (Samarasekara 2008a). In second manuscript, second order perturbed Heisenberg Hamiltonian of ferromagnetic films of five layers with all seven magnetic energy parameters was solved without any special assumptions (Samarasekara and Warnakulasooriya 2016). In addition, the third order perturbed Heisenberg Hamiltonian was solved for thick ferromagnetic films under several special assumptions (Samarasekara $2008 \mathrm{~b})$. The terms with third order of $\varepsilon$ were considered in third order perturbed Heisenberg Hamiltonian. The third order perturbed Heisenberg Hamiltonian with all seven magnetic energy parameters was solved for ferromagnetic films with three layers (Samarasekara and Yapa 2016). Third order perturbed Heisenberg Hamiltonian was also employed to explain the magnetic properties of ferrites (Samarasekara 2014).

The Heisenberg Hamiltonian with few magnetic energy parameters has been employed to explain ferromagnetic materials by some other researchers. Effect of the interfacial coupling on the magnetic ordering in ferro-antiferromagntic bilayers has been studied using the Heisenberg Hamiltonian (Tsai et al. 2003). Heisenberg Hamiltonian with spin exchange interaction, magnetic dipole interaction, applied magnetic field, second and fourth order magnetic anisotropy terms has been solved for ferromagnetic thin films (Hucht and Usadel 1997, Hucht and Usadel 1999, Usadel and Hucht 2002). Computer simulations have been used to 
explain the domain structure and Magnetization reversal in thin magnetic films (Nowak 1995). Heisenberg Hamiltonian was used to describe inplane dipole coupling anisotropy of a square ferromagnetic Heisenberg monolayer (Dantziger et al. 2002). In addition, some other models have been used as following. The quasistatic magnetic hysteresis of ferromagnetic thin films grown on a vicinal substrate has been theoretically investigated using 2-D Monte Carlo simulations (Zhao et al. 2002). Structural and magnetic properties of two dimensional FeCo ordered alloys deposited on W(110) substrates have been determined by first principles band structure theory (Spisak and Hafner 2005). EuTe films with surface elastic stresses have been theoretically studied using Heisenberg Hamiltonian (Radomska and Balcerzak 2003). De Vries theory was employed to explain the magnetostriction of $\mathrm{dc}$ magnetron sputtered
FeTaN thin films (Cates and Alexander 1994). Magnetic layers of $\mathrm{Ni}$ on $\mathrm{Cu}$ have been theoretically investigated using the KorringaKohn-Rostoker Green's function method (Ernst et al. 2000). Electric and magnetic properties of multiferroic thin films have been theoretically described using modified Heisenberg and transverse Ising model combined with Green's function technique (Kovachev and Wesselinowa 2009).

The solution of third order perturbed Heisenberg Hamiltonian for body centered cubic (bcc) structured ferromagnetic films with number of spin layers up to 30 will be presented in this manuscript. All the magnetic energy parameters were included in the solution of third order perturbed Heisenberg Hamiltonian. MATLAB computer software programs were incorporated for these simulations.

\section{MODEL}

The classical Heisenberg Hamiltonian of ferromagnetic thin films can be given in following form.

$$
\begin{aligned}
H= & -\frac{J}{2} \sum_{m, n} \vec{S}_{m} \cdot \vec{S}_{n}+\frac{\omega}{2} \sum_{m \neq n}\left(\frac{\vec{S}_{m} \cdot \vec{S}_{n}}{r_{m n}{ }^{3}}-\frac{3\left(\vec{S}_{m} \cdot \vec{r}_{m n}\right)\left(\vec{r}_{m n} \cdot \vec{S}_{n}\right)}{r_{m n}{ }^{5}}\right)-\sum_{m} D_{\lambda_{m}}{ }^{(2)}\left(S_{m}{ }^{z}\right)^{2}-\sum_{m} D_{\lambda_{m}}{ }^{(4)}\left(S_{m}{ }^{z}\right)^{4} \\
& -\sum_{m, n}\left[\vec{H}-\left(N_{d} \vec{S}_{n} / \mu_{0}\right)\right] \cdot \vec{S}_{m}-\sum_{m} K_{s} \operatorname{Sin} 2 \theta_{m}
\end{aligned}
$$

Above equation will be reduced to following form (Hucht and Usadel 1997, Hucht and Usadel 1999, Usadel and Hucht 2002).

$$
\begin{aligned}
E(\theta)= & -\frac{1}{2} \sum_{m, n=1}^{N}\left[\left(J Z_{|m-n|}-\frac{\omega}{4} \Phi_{|m-n|}\right) \cos \left(\theta_{m}-\theta_{n}\right)-\frac{3 \omega}{4} \Phi_{|m-n|} \cos \left(\theta_{m}+\theta_{n}\right)\right] \\
& -\sum_{m=1}^{N}\left(D_{m}^{(2)} \cos ^{2} \theta_{m}+D_{m}^{(4)} \cos ^{4} \theta_{m}+H_{\text {in }} \sin \theta_{m}+H_{\text {out }} \cos \theta_{m}\right) \\
& +\sum_{m, n=1}^{N} \frac{N_{d}}{\mu_{0}} \cos \left(\theta_{m}-\theta_{n}\right)-K_{s} \sum_{m=1}^{N} \sin 2 \theta_{m}
\end{aligned}
$$

Here $m$ and $n$ represent indices of two different layers, $N$ is the number of layers measured in direction perpendicular to the film plane, $J$ is the magnetic spin exchange interaction, $Z_{|m-n|}$ is 
the number of nearest spin neighbors, $\omega$ is the strength of long range dipole interaction, $\Phi_{|m-n|}$ are constants for partial summation of dipole interaction, $D_{\mathrm{m}}{ }^{(2)}$ and $D_{\mathrm{m}}{ }^{(4)}$ are second and fourth order anisotropy constants, $\mathrm{H}_{\text {in }}$ and $\mathrm{H}_{\text {out }}$ are components of applied magnetic field, $\mathrm{N}_{\mathrm{d}}$ is the demagnetization factor, and $\mathrm{K}_{\mathrm{s}}$ is the constant related to the stress which depends on the magnetization and the magnitude of stress.

For non-oriented films above angles $\theta_{\mathrm{m}}$ and $\theta_{\mathrm{n}}$ measured with film normal can be expressed in forms of $\theta_{m}=\theta+\varepsilon_{m}$ and $\theta_{n}=\theta+\varepsilon_{n}$, and above energy can be expanded up to the third order of $\varepsilon$ as following,

$E(\theta)=E_{0}+E(\varepsilon)+E\left(\varepsilon^{2}\right)+E\left(\varepsilon^{3}\right)$

Here $E_{0}=-\frac{1}{2} \sum_{m, n=1}^{N}\left(J Z_{|m-n|}-\frac{\omega}{4} \Phi_{|m-n|}\right)+\frac{3 \omega}{8} \cos 2 \theta \sum_{m, n=1}^{N} \Phi_{|m-n|}$

$$
-\cos ^{2} \theta \sum_{m=1}^{N} D_{m}^{(2)}-\cos ^{4} \theta \sum_{m=1}^{N} D_{m}^{(4)}-N\left(H_{\text {in }} \sin \theta+H_{\text {out }} \cos \theta-\frac{N_{d}}{\mu_{0}}+K_{s} \sin 2 \theta\right)
$$

$E(\varepsilon)=-\frac{3 \omega}{8} \sin 2 \theta \sum_{m, n=1}^{N} \Phi_{|m-n|}\left(\varepsilon_{m}+\varepsilon_{n}\right)+\sin 2 \theta \sum_{m=1}^{N} D_{m}{ }^{(2)} \varepsilon_{m}+2 \cos ^{2} \theta \sin 2 \theta \sum_{m=1}^{N} D_{m}{ }^{(4)} \varepsilon_{m}$

$-H_{\text {in }} \cos \theta \sum_{m=1}^{N} \varepsilon_{m}+H_{\text {out }} \sin \theta \sum_{m=1}^{N} \varepsilon_{m}-2 K_{s} \cos 2 \theta \sum_{m=1}^{N} \varepsilon_{m}$

$E\left(\varepsilon^{2}\right)=\frac{1}{4} \sum_{m, n=1}^{N}\left(J Z_{|m-n|}-\frac{\omega}{4} \Phi_{|m-n|}\right)\left(\varepsilon_{m}-\varepsilon_{n}\right)^{2}-\frac{3 \omega}{16} \cos 2 \theta \sum_{m, n=1}^{N} \Phi_{|m-n|}\left(\varepsilon_{m}+\varepsilon_{n}\right)^{2}$

$-\left(\sin ^{2} \theta-\cos ^{2} \theta\right) \sum_{m=1}^{N} D_{m}{ }^{(2)} \varepsilon_{m}{ }^{2}+2 \cos ^{2} \theta\left(\cos ^{2} \theta-3 \sin ^{2} \theta\right) \sum_{m=1}^{N} D_{m}{ }^{(4)} \varepsilon_{m}{ }^{2}$

$+\frac{H_{i n}}{2} \sin \theta \sum_{m=1}^{N} \varepsilon_{m}{ }^{2}+\frac{H_{\text {out }}}{2} \cos \theta \sum_{m=1}^{N} \varepsilon_{m}{ }^{2}-\frac{N_{d}}{2 \mu_{0}} \sum_{m, n=1}^{N}\left(\varepsilon_{m}-\varepsilon_{n}\right)^{2}$

$+2 K_{s} \sin 2 \theta \sum_{m=1}^{N} \varepsilon_{m}{ }^{2}$

$E\left(\varepsilon^{3}\right)=\frac{\omega}{16} \sin 2 \theta \sum_{m, n=1}^{N}\left(\varepsilon_{m}+\varepsilon_{n}\right)^{3} \phi_{|m-n|}-\frac{4}{3} \cos \theta \sin \theta \sum_{m=1}^{N} D_{m}{ }^{(2)} \varepsilon_{m}{ }^{3}$

$-4 \cos \theta \sin \theta\left(\frac{5}{3} \cos ^{2} \theta-\sin ^{2} \theta\right) \sum_{m=1}^{N} D_{m}{ }^{(4)} \varepsilon_{m}{ }^{3}+\frac{H_{i n}}{6} \cos \theta \sum_{m=1}^{N} \varepsilon_{m}{ }^{3}$

$-\frac{H_{\text {out }}}{6} \sin \theta \sum_{m=1}^{N} \varepsilon_{m}{ }^{3}+\frac{4 K_{s}}{3} \cos 2 \theta \sum_{m=1}^{N} \varepsilon_{m}{ }^{3}$

After using the constraint $\sum_{m=1}^{N} \varepsilon_{m}=0, \mathrm{E}(\varepsilon)=\vec{\alpha} \cdot \vec{\varepsilon}$

Here $\vec{\alpha}(\varepsilon)=\vec{B}(\theta) \sin 2 \theta$ are the terms of matrices with 
$B_{\lambda}(\theta)=-\frac{3 \omega}{4} \sum_{m=1}^{N} \Phi_{|\lambda-m|}+D_{\lambda}^{(2)}+2 D_{\lambda}^{(4)} \cos ^{2} \theta$

Also $E\left(\varepsilon^{2}\right)=\frac{1}{2} \vec{\varepsilon} \cdot C \cdot \vec{\varepsilon}$

Here the elements of matrix $\mathrm{C}$ can be given as following,

$$
\begin{aligned}
C_{m n} & =-\left(J Z_{|m-n|}-\frac{\omega}{4} \Phi_{|m-n|}\right)-\frac{3 \omega}{4} \cos 2 \theta \Phi_{|m-n|}+\frac{2 N_{d}}{\mu_{0}} \\
& +\delta_{m n}\left\{\sum_{\lambda=1}^{N}\left[J Z_{|m-\lambda|}-\Phi_{|m-\lambda|}\left(\frac{\omega}{4}+\frac{3 \omega}{4} \cos 2 \theta\right)\right]-2\left(\sin ^{2} \theta-\cos ^{2} \theta\right) D_{m}{ }^{(2)}\right. \\
& \left.+4 \cos ^{2} \theta\left(\cos ^{2} \theta-3 \sin ^{2} \theta\right) D_{m}{ }^{(4)}+H_{\text {in }} \sin \theta+H_{\text {out }} \cos \theta-\frac{4 N_{d}}{\mu_{0}}+4 K_{s} \sin 2 \theta\right\}
\end{aligned}
$$

Also $E\left(\varepsilon^{3}\right)=\varepsilon^{2} \beta . \vec{\varepsilon}$

Here matrix elements of matrix $\beta$ can be given as following,

$$
\begin{aligned}
\beta_{m n}= & \frac{3 \omega}{8} \sin 2 \theta \Phi_{|m-n|}+\delta_{m n}\left\{\frac{\omega}{8} \sin 2 \theta\left[A_{m}-\Phi_{0}\right]-\frac{4}{3} \cos \theta \sin \theta D_{m}{ }^{(2)}\right. \\
& -4 \cos \theta \sin \theta\left(\frac{5}{3} \cos ^{2} \theta-\sin ^{2} \theta\right) D_{m}{ }^{(4)}+\frac{H_{\text {in }}}{6} \cos \theta-\frac{H_{\text {out }}}{6} \sin \theta \\
& \left.+\frac{4 K_{s}}{3} \cos 2 \theta\right\}
\end{aligned}
$$

Also $\beta_{n m}=\beta_{m n}$ and matrix $\beta$ is symmetric.

Here $A_{m}$ values are different for even and odd $\mathrm{N}$ values, and can be given as following.

For odd $N, A_{\frac{N}{2}+0.5+n}=2 \sum_{v=0}^{\frac{N}{2}-0.5-n} \Phi_{v}+\sum_{v=\frac{N}{2}+0.5-n}^{\frac{N}{2}+n-0.5} \Phi_{v}$ for $m>\frac{N}{2}$,

where $n$ varies from 1 to $\frac{N}{2}-0.5$.

When $n=0, A_{\frac{N}{2}+0.5+n}=2 \sum_{v=0}^{\frac{N}{2}-0.5-n} \Phi_{v}$

$A_{\mathrm{m}}$ for $m<\frac{N}{2}$ can be obtained using $A_{\frac{N}{2}+0.5+n}=A_{\frac{N}{2}+0.5-n}$

For even $N, A_{\frac{N}{2}+1+n}=2 \sum_{v=0}^{\frac{N}{2}-1-n} \Phi_{v}+\sum_{v=\frac{N}{2}-n}^{\frac{N}{2}+n} \Phi_{v}$ for $m>\frac{N}{2}$, 
where n varies from 0 to $\frac{N}{2}-1$.

$A_{\mathrm{m}}$ for $m<\frac{N}{2}$ can be obtained using $A_{\frac{N}{2}+1+n}=A_{\frac{N}{2}-n}$

Therefore, the total magnetic energy given in equation 2 can be deduced to

$E(\theta)=E_{0}+\vec{\alpha} \cdot \vec{\varepsilon}+\frac{1}{2} \vec{\varepsilon} \cdot C \cdot \vec{\varepsilon}+\varepsilon^{2} \beta \cdot \vec{\varepsilon}$

Because the derivation of a final equation for $\varepsilon$ with the third order of $\varepsilon$ in above equation is tedious, only the second order of $\varepsilon$ will be considered for following derivation.

Then $E(\theta)=\mathrm{E}_{0}+\vec{\alpha} \cdot \vec{\varepsilon}+\frac{1}{2} \vec{\varepsilon} \cdot C \cdot \vec{\varepsilon}$

Using a suitable constraint in above equation, it is possible to show that $\vec{\varepsilon}=-C^{+} . \vec{\alpha}$

Here $C^{+}$is the pseudo-inverse given by

$$
C . C^{+}=1-\frac{E}{N} \text {. }
$$

$E$ is the matrix with all elements given by $E_{\mathrm{mn}}=1$.

After using $\varepsilon$ in equation $7, E(\theta)=E_{0}-\frac{1}{2} \vec{\alpha} \cdot C^{+} \cdot \vec{\alpha}-\left(C^{+} \alpha\right)^{2} \vec{\beta}\left(C^{+} \alpha\right)$

\section{RESULTS AND DISCUSSION}

The magnetic dipole interactions for complicated ferromagnetic structures such as cobalt were determined by calculating the dipole interactions between each pair of spin within the lattice (Samarasekara and Ariyaratne 2012). However, the magnetic dipole interactions for simple lattice structures such as sc and bcc can be expressed in constants arising from partial summations of dipole interaction $\left(\Phi_{|m-n|}\right)$ (Hucht and Usadel 1997, Hucht and Usadel 1999, Usadel and Hucht 2002). The magnetic dipole interaction is the microscopic level interaction between spins. However, the demagnetization factor is the macroscopic level interactions between spins. Therefore, both magnetic dipole interaction and demagnetization factor were taken into account. Demagnetization factor depends on the size and the shape of the sample. In S.I units, demagnetization factor $\left(N_{d}\right)$ perpendicular to the film plane is 1 , and the value of $N_{d}$ in the film plane is 0 .

First $\vec{\alpha}$ and $C$ matrix elements were found using equations 4 and 5. Then $\beta$ and $C^{+}$matrix elements were found using equations 6 and 8 . Finally total magnetic energy was found using equation 9 for different values of $\frac{J}{\omega}, \frac{H_{i n}}{\omega}, \frac{N_{d}}{\mu_{0} \omega}, \frac{H_{o u t}}{\omega}, \frac{K_{s}}{\omega}, \frac{D_{m}^{(2)}}{\omega}$ and $\frac{D_{m}^{(4)}}{\omega}$. Because all $J, \omega, H_{\text {in }}, H_{\text {out }}, N_{d} / \mu_{0}, K_{s}, D_{m}^{(2)}$ and $D_{m}{ }^{(4)}$ have the same dimensions, all $\frac{J}{\omega}, \frac{H_{i n}}{\omega}, \frac{N_{d}}{\mu_{0} \omega}, \frac{H_{\text {out }}}{\omega}, \frac{K_{s}}{\omega}, \frac{D_{m}{ }^{(2)}}{\omega}$ and $\frac{D_{m}{ }^{(4)}}{\omega}$ are dimensionless quantities.

Figure 1 shows the 3-D plot of energy versus stress induced anisotropy and the azimuthal angle for bcc structure with 30 spin layers $(\mathrm{N}=30)$. For bcc (001) lattice $\mathrm{Z}_{0}=0, \mathrm{Z}_{1}=4$, $\mathrm{Z}_{2}=0, \Phi_{0}=5.8675$ and $\Phi_{1}=2.7126$ (Hucht and Usadel 1997, Hucht and Usadel 1999, Usadel and Hucht 2002). Other parameters were kept at $\frac{J}{\omega}=\frac{H_{\text {in }}}{\omega}=\frac{N_{d}}{\mu_{0} \omega}=\frac{H_{\text {out }}}{\omega}=10, \frac{D_{m}{ }^{(2)}}{\omega}=30$ and $\frac{D_{m}{ }^{(4)}}{\omega}=20$. Several close packed energy minimums and maximums can be observed in 
this case. On the other hand, the peaks of the same 3-D plot of energy versus stress induced anisotropy and angle were widely spread for simple cubic structured ferromagnetic films with four spin layers (Samarasekara 2008a). The magnetic properties of thin films depend on stress induced anisotropy (Naoto 1989). The coercivity also varies with the deposition temperature of thin films due to stress induced anisotropy. The stress induced anisotropy depends on the thermal expansion coefficients of magnetic thin film material and the substrate. In addition, it depends on the Young's modulus and the Poisson's ratio of the magnetic material.

Shape of the same 3-D plots of energy versus stress induced anisotropy and angle for the fcc structured ferromagnetic films with five spin layers was entirely different form the 3-D plots in this manuscript (Samarasekara and Warnakulasooriya 2016). The $2^{\text {nd }}$ order perturbed Heisenberg Hamiltonian was applied to find the magnetic energy of four spin layered and five spin layered films ((Samarasekara 2008a, Samarasekara and Warnakulasooriya 2016). The third order perturbed Heisenberg Hamiltonian was solved for fcc structured ferromagnetic films with three spin layers (Samarasekara and Yapa 2016). The peaks were less spaced in that case too. In addition, although the total magnetic energy of films with three spin layers varies up to $10^{4}$ (Samarasekara and Yapa 2016), that of films with 30 spin layers changes up to $10^{7}$ according to figure 1 . This is attributed to the fact that the total magnetic energy of films simply increases with the number of spin layers. For thick ferromagnetic films with 1000 spin layers, shapes of 3-D plots obtained using third order perturbed Heisenberg Hamiltonian were entirely different (Samarasekara 2008b).

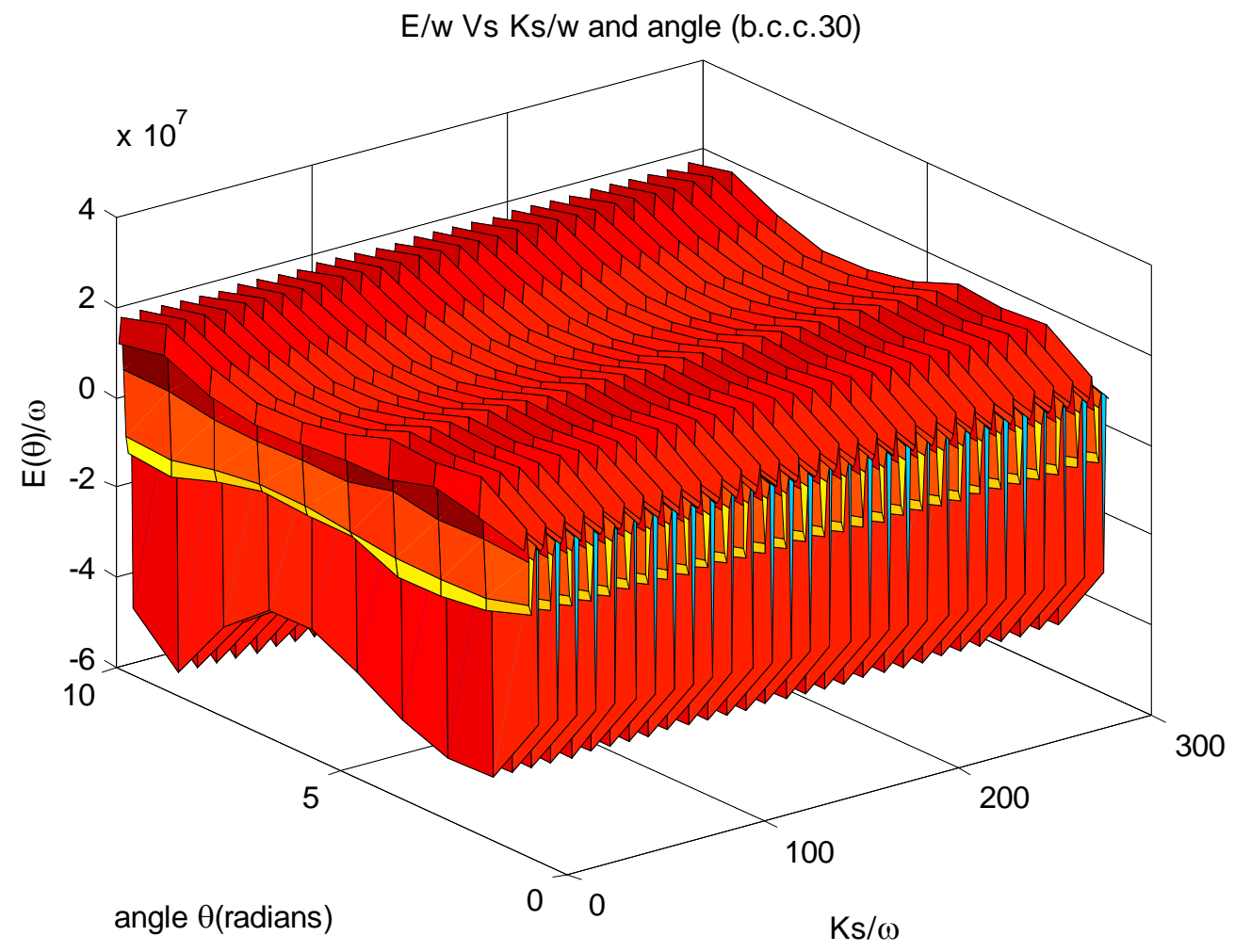

Figure 1: 3-D plot of $\frac{E(\theta)}{\omega}$ versus angle and $\frac{K_{s}}{\omega}$ for bcc structure. 
A minima of 3-D plot in figure 1 can be observed at $\frac{K_{s}}{\omega}=8$. Figure 2 shows the graph of $\frac{E(\theta)}{\omega}$ versus angle at $\frac{K_{s}}{\omega}=8$. A minimum and a maximum of this plot can be observed at $10.8002^{\circ}$ and $115.2219^{\circ}$, respectively. So the magnetic easy direction can be observed at $10.8002^{0}$.

A maxima of 3-D plot in figure 1 can be observed at $\frac{K_{s}}{\omega}=2$. Figure 3 shows the graph of $\frac{E(\theta)}{\omega}$ versus angle at $\frac{K_{s}}{\omega}=2$. A minimum and a maximum of this plot can be observed at $5.4001^{0}$ and $108.0026^{\circ}$, respectively. So the magnetic hard direction can be observed at $108.0026^{\circ}$.

Figure 4 shows the 3 -D plot of $\frac{E(\theta)}{\omega}$ versus angle and $\frac{J}{\omega}$ for $\mathrm{N}=30$. Other parameters were kept at $\frac{H_{\text {in }}}{\omega}=\frac{N_{d}}{\mu_{0} \omega}=\frac{H_{\text {out }}}{\omega}=\frac{K_{s}}{\omega}=10$, $\frac{D_{m}{ }^{(2)}}{\omega}=30$ and $\frac{D_{m}{ }^{(4)}}{\omega}=20$ A minima and a maxima of this 3-D plot can be observed at $\frac{J}{\omega}=7$ and 18 , respectively. By plotting the graph

of $\frac{E(\theta)}{\omega}$ versus angle at $\frac{J}{\omega}=7$, energy minima and maxima were found to be at $12.5993^{\circ}$ and $116.9980^{\circ}$. So the magnetic easy direction is $12.5993^{0}$. By plotting the graph of $\frac{E(\theta)}{\omega}$ versus angle at $\frac{J}{\omega}=18$, energy minima and maxima were found to be at $12.5993^{\circ}$ and $116.9980^{\circ}$. So the magnetic hard direction is $116.9980^{\circ}$. In this case, the magnetic easy and hard directions are independent of the value of $\frac{J}{\omega}$. The shape of the same 3-D plot of energy versus angle and spin exchange interaction for the fcc structured ferromagnetic films with three spin layers obtained using third order perturbed Heisenberg Hamiltonian was entirely different (Samarasekara and Yapa 2016). Although the total magnetic energy of films with three spin layers varies up to 100 , that of films with 30 spin layers changes up to $10^{4}$. This implies that the total magnetic energy gradually increases with the number of spin layers.

Then the magnetic easy and hard directions were found for the films by plotting graphs of energy versus angle at $\frac{H_{\text {in }}}{\omega}=\frac{J}{\omega}=\frac{N_{d}}{\mu_{0} \omega}=\frac{H_{\text {out }}}{\omega}=\frac{K_{s}}{\omega}=\frac{D_{m}{ }^{(2)}}{\omega}=\frac{D_{m}{ }^{(4)}}{\omega} 10$ for different values of spin layers. Table 1 shows the variation of magnetic easy directions and the magnetic energy from 10 to 30 spin layers. Because $\theta$ is the angle of easy direction measured from a line drawn perpendicular to the film plane, magnetic easy direction gradually rotates from out of plane to in plane direction. Experimental data also varifies that the easy axis of ferromagnetic thin films rotates from out of plane to in plane direction with the increase of the thickness. According to the data of sputtered ferromagnetic $\mathrm{Ni}$ films, a preferred in plane orientation of magnetic easy axis can be observed at higher thicknesses (Parlak et al. 2015). The spin reorientation transition occurs from in plane to out plane at film thickness of 14-24 ${ }^{0} \mathrm{~A}$. According to the experimental data of ferromagnetic $\mathrm{Fe}$ thin films fabricated by electron beam evaporation, the magnetic easy axis indicates a preferred in plane orientation above thicknesses of 2 monolayers and out of plane orientation below 2 monolayers (ArayaPochet et al. 1988). 


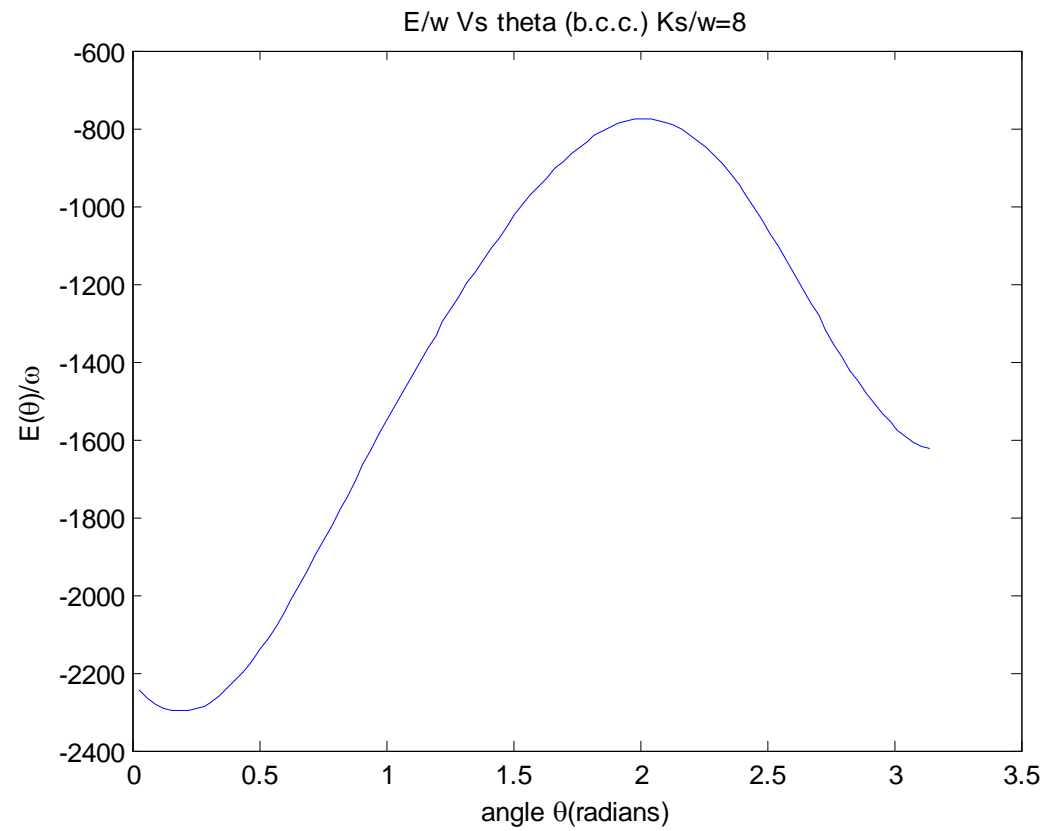

Figure 2: $\frac{E(\theta)}{\omega}$ versus angle at $\frac{K_{s}}{\omega}=8$ for $\mathrm{N}=30$.

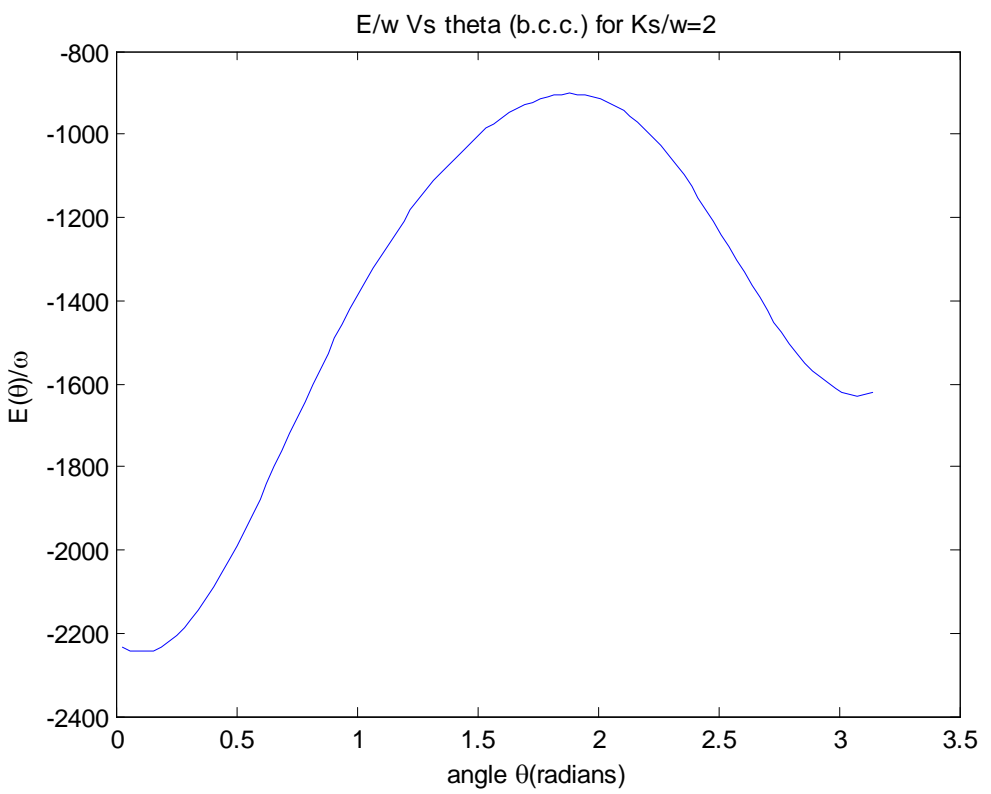

Figure 3: $\frac{E(\theta)}{\omega}$ versus angle at $\frac{K_{s}}{\omega}=2$ for $\mathrm{N}=30$ 


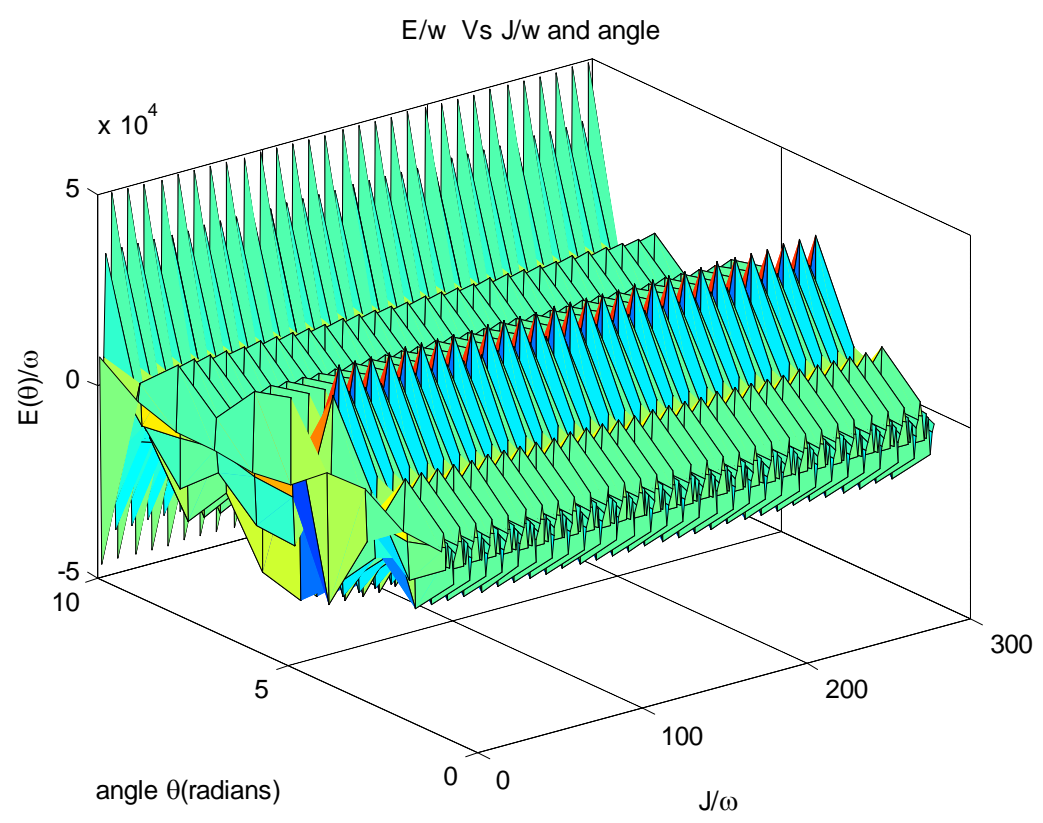

Figure 4: 3-D plot of $\frac{E(\theta)}{\omega}$ versus angle and $\frac{J}{\omega}$ for bec structure.

Table 1: Variation of magnetic easy direction with number of spin layers.

\begin{tabular}{llllll}
\hline $\begin{array}{l}\text { Number of spin } \\
\text { layers }\end{array}$ & $\begin{array}{l}\theta \text { (easy) in } \\
\text { degrees }\end{array}$ & $E / \omega$ (easy) & $E / \omega$ (hard) & $\begin{array}{l}\Delta \mathrm{E}=E / \omega(\text { hard }) \\
-E / \omega(\text { easy) }\end{array}$ & $\begin{array}{l}\Delta \theta=\theta(\text { hard }) \\
\theta(\text { easy) in } \\
\text { degrees }\end{array}$ \\
\hline 10 & 28.8026 & -512.4 & -154.2 & 358.2 & 108.0198 \\
\hline 20 & 30.6017 & -1035 & -319.9 & 715.1 & 106.2207 \\
\hline 30 & 30.6017 & -1558 & -485.8 & 1072.2 & 106.2207 \\
\hline
\end{tabular}

According to table 1, the energy $(\Delta \mathrm{E})$ required to rotate spins from easy to hard (or vice versa) direction gradually increases with the number of spin layers. The higher the $\Delta \mathrm{E}$ is the higher the magnetic anisotropy. The films with higher or smaller $\Delta \mathrm{E}$ values are useful in the applications of hard or soft magnetic materials, respectively. The angle between easy and hard directions slightly changes with the number of spin layers, and it is fairly close to 90 degrees. According to our previous data obtained using $2^{\text {nd }}$ order Heisenberg Hamiltonian, the magnetic energy along easy and hard directions was also negative (Samarasekara 2008a). In addition, the angle between easy and hard directions obtained using the third order perturbation in this manuscript is fairly close to the angle between easy and hard directions obtained using the second order perturbation in one of our previous manuscripts
Warnakulasooriya 2016). The negative value of energy along easy and hard directions gradually increases with the number of layers. Spin exchange interaction, second order magnetic anisotropy, fourth order magnetic anisotropy, magnetic field energy and stress induced magnetic anisotropy contribute to the negative values of the total magnetic energy, according to the Heisenberg Hamiltonian equation given in this manuscript. On the other hand, magnetic dipole interaction and the demagnetization factor contribute to the positive values of total magnetic energy. This implies that the spin exchange interaction, second order magnetic anisotropy, fourth order magnetic anisotropy, magnetic field energy and stress induced magnetic anisotropy becomes dominant at higher thicknesses of ferromagnetic films.

The easy axis orientation of magnetic thin films mainly depends on the substrate 
temperature and orientation of the substrate. In sputtering techniques, easy axis orientation depends on the deposition rate, thickness of the film, sputtering pressure, type of sputtering gas, distance between substrate and target, and annealing conditions (Cadieu 1986a, Cadieu 1986b, Kamprath 1998). Variation of magnetic easy direction with deposition temperature was theoretically explained using Heisenberg Hamiltonian by us (Samarasekara and Gunawardhane 2011, Samarasekara and Saparamadu Udara 2012, Samarasekara and Saparamadu 2013a, Samarasekara and Saparamadu 2013b). The idea of spin reorientation was incorporated with the variation of the average value of one of the spin components in 2-D model in that simulation.

\section{CONCLUSION}

One of the energy minimums and maximums of this 3-D plot were observed at $\frac{K_{s}}{\omega}=8$ and 2 , respectively. According to graphs of energy versus angle, the magnetic easy and hard directions corresponding to these $\frac{K_{s}}{\omega}$ values were found to be $10.8002^{\circ}$ and $108.0026^{0}$, respectively. Similarly by plotting 3-D graph of energy versus spin exchange interaction and angle, one of the energy minimums and maximums could be found at $\frac{J}{\omega}=7$ and 18 , respectively. Magnetic easy and hard directions related to the plot of $\frac{J}{\omega}$ were found to be $12.5993^{\circ}$ and $116.9980^{\circ}$. The magnetic easy axis gradually rotates from perpendicular to in plane direction of the film plane, as the number of spin layers is increased. Our theoretical data agree with the experimental data obtained for ferromagnetic $\mathrm{Ni}$ and $\mathrm{Fe}$ thin films by some other researchers. The energy required to rotate spins from easy direction to hard direction gradually increases with the number of spin layers. Because the total number of spins in the film increases with the number of spin layers, the energy required to rotate spins also increases with the number of spin layers. The negative value of energy along easy direction gradually increases with the number of spin layers. However, the angle between easy and hard directions changes slightly.

\section{REFERENCES}

Araya-Pochet J, Ballentine C.A and Erskine J.L. (1988). Thickness and temperature dependent spin anisotropy of ultrathin epitaxial Fe films on Ag(100). Physical Review B 38(11): 7846-7849

Cadieu F.J, Cheung T.D and Wickramasekara L. (1986a). Magnetic properties of sputtered Nd-FeB films. Journal of Magnetism and Magnetic materials 54: 535-536.

Cadieu F.J, Cheung T.D and Wickramasekara L. (1986b). Magnetic properties of Sm-Ti-Fe and Sm-Co based films. Journal of Applied Physics 57(8): 4161-4163.

Cates James C. and Alexander Jr Chester. (1994). Theoretical study of magnetostriction in FeTaN thin films. Journal of Applied Physics 75: 67546756.

Dantziger M, Glinsmann B, Scheffler S, Zimmermann B and Jensen P.J. (2002). In-plane dipole coupling anisotropy of a square ferromagnetic Heisenberg monolayer. Physical Review B 66: 094416.

Ernst A, Lueders M, Temmerman W.M, Szotek Z and Van der Laan G. (2000). Theoretical study f magnetic layers of nickel on copper; dead or live?. Journal of Physics: Condensed matter 12(26): 5599-5606.

Hucht A and Usadel K.D. (1997). Reorientation transition of ultrathin ferromagnetic films. Physical Review B 55: 12309.

Hucht A and Usadel K.D. (1999). Theory of the spin reorientation transition of ultra-thin ferromagnetic films. Journal of Magnetism and Magnetic materials 203(1): 88-90.

Kamprath N, Liu N.C, Hegde H and Cadieu F.J. (1998). Magnetic properties and synthesis of high ${ }_{\mathrm{i}} \mathrm{H}_{\mathrm{c}} \mathrm{Sm}-\mathrm{Ti}-\mathrm{Fe}$. Journal of Applied Physics 64(10): 10.1063.

Kovachev St and Wesselinowa J.M. (2009). Theoretical study of multiferroic thin films based on a microscopic model. Journal of Physics: Condensed matter 21(22): 225007.

Naoto Sugimoto, Takashi Inukai, Morito Matsuoka and Ken'ichi Ono (1989). Stress induced perpendicular magnetic anisotropy in PtMnSb thin films. Japanese Journal of Applied Physics 28(1): 1139-1140.

Nowak U. (1995). Magnetisation reversal and domain structure in thin magnetic films: Theory and computer simulation. IEEE transaction on magnetics 31(6-2): 4169-4171.

Parlak U, Akoz M.E, Tokdemir Ozturk S and Erkovan M. (2015). Thickness dependent magnetic properties of polycrystalline nickel thin films. Acta Physica Polonica A 127(4): 995-997.

Radomska Anna and Balcerzak Tadeusz (2003). Theoretical studies of model thin EuTe films with 
surface elastic stresses. Central European Journal of Physics 1(1): 100-117.

Samarasekara P. (2008a). Four layered ferromagnetic ultra-thin films explained by second order perturbed Heisenberg Hamiltonian. Ceylon Journal of Science 14: 11-19.

Samarasekara P. (2008b). Influence of third order perturbation on Heisenberg Hamiltonian of thick ferromagnetic films. Electronic Journal of Theoretical Physics 5(17): 227-236.

Samarasekara P and Gunawardhane N.H.P.M. (2011). Explanation of easy axis orientation of ferromagnetic films using Heisenberg Hamiltonian. Georgian electronic scientific journals: Physics 2(6): 62-69.

Samarasekara P and Saparamadu Udara (2012). Investigation of Spin Reorientation in Nickel Ferrite Films. Georgian electronic scientific journals: Physics 1(7): 15-20.

Samarasekara P and Ariyaratne Amila D. (2012). Determination of magnetic properties of Cobalt films using second order perturbed Heisenberg Hamiltonian. Research \& Reviews: Journal of Physics-STM journals 1(1): 16-23.

Samarasekara P and Saparamadu Udara (2013a). In plane oriented Strontium ferrite thin films described by spin reorientation. Research \& Reviews: Journal of Physics-STM journals 2(2): 12-16.

Samarasekara P and Saparamadu Udara (2013b). Easy axis orientation of barium hexa-ferrire films as explained by spin reorientation. Georgian electronic scientific journals: Physics 1(9): 1015.

Samarasekara P. (2014). Cobalt ferrite films as described by third order perturbed Heisenberg Hamiltonian. Journal of science: University of Kelaniya 9: 27-38.

Samarasekara P and Warnakulasooriya B.I. (2016). Five layered fcc ferromagnetic films as described by modified second order perturbed Heisenberg Hamiltonian. Journal of science: University of Kelaniya 11: 11-21.

Samarasekara P and Yapa N.U.S. (2016). Third order perturbed energy of fcc ferromagnetic thin films as described by Heisenberg Hamiltonian. Ceylon Journal of Science 45(2): 71-77.

Spisak D and Hafner J. (2005). Theoretical study of $\mathrm{FeCo} / \mathrm{W}(110)$ surface alloys. Journal of Magnetism and Magnetic Materials 286: 386389.

Tsai Shan-Ho, Landau D.P and Schulthess Thomas C. (2003). Effect of interfacial coupling on the magnetic ordering in ferro-antiferromagntic bilayers. Journal of Applied Physics 93(10): 8612-8614.

Usadel K.D and Hucht A. (2002). Anisotropy of ultrathin ferromagnetic films and the spin reorientation transition. Physical Review $B$ 66: 024419.

Zhao D, Feng Liu, Huber D.L and Lagally M.G. (2002). Step-induced magnetic-hysteresis anisotropy in ferromagnetic thin films. Journal of Applied Physics 91(5): 3150-3153. 